\title{
The Effect of Heat on the Ability of a Host Strain to Support the Growth of a Staphylococcus Phage
}

\author{
By ELIZABETH A. ASHESHOV and M. PATRICIA JEVONS \\ Staphylococcus Reference Laboratory, Central Public Health Laboratory, \\ Colindale, London, N.W. 9
}

(Received 9 August 1962)

\begin{abstract}
SUMMARY
A group of epidemiologically related strains of Staphylococcus aureus, which were untypable by standard methods, became sensitive to typing phage 187 after the inocula had been held at $56^{\circ}$ for $2 \mathrm{~min}$. The strains were found to be lysogenic. Evidence is presented to show that the phage carried, phage 5504', was exerting interference against phage 187 which could be partially overcome by heat treatment. Phage 187 grown in heated cocci was found to have undergone a host-induced modification which rendered it capable of lysing unheated lysogenic organisms. Active multiplication of the carried phage, phage 5504', appeared to be necessary for the multiplication of phage 187. Ultraviolet irradiation also increased the sensitivity of the strain to phage 187, but to a lesser degree than heat treatment. It is suggested that the effect of heating is to induce active multiplication of phage 5504' in the lysogenic culture, possibly by destroying a cytoplasmic substance responsible for maintaining the carried phage in its prophage state.
\end{abstract}

\section{INTRODUCTION}

A collection of 14 epidemiologically related coagulase-positive staphylococci was received for bacteriophage typing from Professor Monaci of the Institute of Hygiene, The University of Parma, Italy, in the early part of 1961. These strains, which had been isolated from patients suffering from post-influenzal pneumonia in an oldpeople's home, were described by Muzzetto et al. (1961). They were not typable with the basic set of phages at routine test dilution ( $\mathrm{rtd})$ or at $1000 \times \mathrm{rtd}$. Epidemics due to completely untypable strains of staphylococci are rare in our experience, and an extensive search was made amongst the experimental phages maintained in this laboratory in an attempt to find one which would lyse the strains or could be adapted for this purpose. No such phage was found. A report on the effect of heat on the typing pattern of strains of staphylococci by Ma \& Mandle (1961) led us to repeat the typing of these strains after heating the inocula for $2 \mathrm{~min}$. at $56^{\circ}$. After this treatment all strains were lysed by phage 187 at $1000 \mathrm{rtd}$. Phage 187 was originally designated phage $735 \mathrm{~A}$ by Wahl \& Fouace (1954) and is of interest because it is serologically distinct from all known staphylococcal phages, and strains lysed by this phage have never been found to be lysed by any other phage. Experiments to be described showed that the resistance of these strains to phage 187 was not due to failure of the unheated inocula to absorb the phage, but to interference exerted by a prophage carried by the strains. Heating appeared to overcome this 
interference sufficiently to allow a limited multiplication of phage 187. The phage which grew on such heated inocula was found to be a host-induced modification of phage 187 which was able to multiply in the unheated strain. This phage will be referred to subsequently as modified phage 187 or $187 \mathrm{~m}$.

\section{METHODS}

Strains of Staphylococcus aureus used. (1) 14 strains of $S$. aureus from Professor Monaci. These strains behaved identically in that they became sensitive to phage 187 only after heating, and all carried an apparently identical temperate phage. The majority of experiments were carried out with one of them, strain 5504. (2) The propagating strain (PS) of phage 187, referred to as PS 187 and two lysogenic derivatives of this strain which will be described in the text.

Phage preparations. Phage 187 was grown on its propagating strain Ps 187 by the agar layer method (Swanstrom \& Adams, 1951). Phage 5504', the phage carried by strain 5504, was originally prepared by filtering a broth culture of strain 5504, and subsequently by propagation of the phage on PS 187 . Phage $187 \mathrm{~m}$, the host-range modification of phage 187 resulting from growth in heated cocci of strain 5504, was first prepared by isolating the plaques produced on a lawn arising from a heat-treated inoculum of strain 5504. Later preparations were made by propagating the phage in unheated cocci of strain 5504. All preparations of this phage contained some particles of phage 5504' released by the lysogenic propagating strain but the proportion of phage 5504' in preparations of phage $187 \mathrm{~m}$ did not exceed 1 in $10^{3}$ particles.

Phage antisera. Antisera against phages 187 and 5504' were prepared in rabbits and tested in the usual way (Adams, 1950).

Heat treatment of staphylococcal strains. The most consistent results were produced by washing off the growth of an $18 \mathrm{hr}$. agar slope of the organism with $0.5 \mathrm{ml}$. broth to obtain a heavy suspension; $0.06 \mathrm{ml}$. of this suspension was added to $2 \mathrm{ml}$. nutrient broth previously warmed to $56^{\circ}$; this temperature was maintained for 2 min., after which the tubes were rapidly cooled and the suspension used to flood plates.

Tests for lysogenicity. Strains were tested for lysogenicity by spotting the supernatant fluid of a $4 \mathrm{hr}$. broth culture of the organism on indicator strains. The carried phage was given the same number as the strain from which it was derived, with the prime sign to distinguish it from the strain, i.e. phage 5504' is the phage derived from strain 5504, the convention first proposed by Anderson \& Felix (1953).

Test for non-lysogenic mutants. The lysogenic culture, in a dilution high enough to yield single colonies, was spread on agar plates. After overnight incubation at $37^{\circ}$ these plates were replicated to plates previously flooded with a sensitive indicator strain (Lederberg \& Lederberg, 1952). After incubation at $30^{\circ}$ overnight each lysogenic colony on the replica plate was surrounded by a zone of lysis.

Lysogenization. Strains were lysogenized by spotting a sterile filtrate of the phage on to a lawn of organisms. The plates were incubated overnight at $30^{\circ}$, after which the secondary growth in the area of lysis was plated on an agar plate and, after incubation, colonies were tested for lysogenicity. A strain was considered to be lysogenic when it could be shown to be resistant to the lysogenizing phage, and to 
carry that phage. The lysogenized strains are described in the usual way, i.e. PS 187(5504') indicates PS 187 lysogenized by the phage from strain 5504 and PS 187(187) indicates PS 187 lysogenized by phage 187 .

Efficiency of plating. The efficiency of plating (eop) of each of the three phages described in this paper is a measure of its titre on a given strain relative to its titre on PS 187 where in every case the titre was maximum or eop $=1$.

\section{RESULTS}

All these epidemiologically related strains from Professor Monaci were resistant to the phages maintained in the Staphylococcus Reference Laboratory, even when tested at concentrations 10-100 times stronger than those used in routine techniques. After the inocula had been held at $56^{\circ}$ for 2 min. all strains were lysed by phage 187 at $1000 \mathrm{rtd}$. A preparation of phage 187 containing $5 \times 10^{9}$ particles $/ \mathrm{ml}$, when titrated on PS 187, was tested on heated and unheated suspensions of strain 5504 . A few small turbid plaques could be seen in the area where undiluted phage had been applied to the unheated cocci, representing a titre of about $2.5 \times 10^{2} / \mathrm{ml}$. The titre on the heated cocci was $2.5 \times 10^{5}$. Heating had therefore increased the sensitivity of the strain to the phage a thousand fold and the eop of phage 187 was about $10^{-4}$ on the heated cocci and $10^{-7}$ on the unheated cocci. Sensitivity to phage 187 was gradually lost on subculture so that after 5 daily subcultures in broth the strain was again untypable.

The fact that heat treatment altered the cocci rather than the suspending medium was demonstrated when heated and unheated broth cultures were spun and their supernatant fluids exchanged. The heated cocci suspended in unheated supernatant fluid were sensitive to phage $\mathbf{1 8 7}$ while the unheated cocci in heated supernatant fluid remained insusceptible. The explanation of this phenomenon suggested by Ma \& Mandle (1961) was failure on the part of the strains to absorb the phage. However, adsorption of phage 187 to unheated cocci of strain 5504 was as rapid as to heated cocci and more rapid than its absorption to PS 187 .

\section{Host-induced modification of phage 187 resulting from growth in heated cocci}

Phage 187 had an eop of about $10^{-4}$ on heated cocci of strain 5504. This low efficiency suggested either that these plaques arose from mutant particles present in the stocks of phage 187 , or from particles of phage 187 which had undergone hostinduced modification. When the phage grown in heated cocci was examined it was found to be capable of lysing heated and unheated cocci with an equal eop of about $10^{-1}$. It reverted to its original host range when subsequently propagated on PS 187 and lysed unheated cocci with an eop of $10^{-7}$ and heated cocci with an eop of $10^{-4}$. These facts ruled out the possibility that phage 187 propagated on heated cocci of strain 5504 (phage $187 \mathrm{~m}$ ) represented a host-range mutant of phage 187 and indicated that host-induced modification was responsible for the alteration of host range. This phenomenon was fully described by Luria (1953) and is known to occur in Salmonella typhi (Anderson \& Felix, 1952), Escherichia coli (Luria \& Human, 1952; Bertani \& Weigle, 1953) and in staphylococci (Ralston \& Krueger, 1952). 


\section{Lysogenicity of the strains}

PS 187 was tested for lysogenicity on a number of indicator strains and was found to carry a phage, phage $187^{\prime}$, which was serologically identical with typing phage 187. However, strain 5504 and the other epidemiologically related strains were completely resistant to this phage both before and after heat treatment, and the existence of phage $187^{\prime}$ did not appear to have any bearing on the phenomenon under investigation.

Strain 5504 and the other strains from this outbreak, on the other hand, were found to carry a phage, 5504', which lysed Ps 187 and all 25 other staphylococcal strains of phage type 187 which were tested. Phage 5504' did not lyse any other indicator strain tested. This specificity suggested that it might be closely related to phage 187 . However, 5504' was not neutralized by antiserum prepared against phage 187 and did not belong to any of the known serological groups.

Antiserum was prepared against phage 5504'. This serum, and antiserum prepared against phage 187, were tested against the three phages, 187, $187 \mathrm{~m}$ and 5504'. Antiserum against phage 187, at a dilution of $1 / 1000$, neutralized about $90 \%$ of both phages 187 and $187 \mathrm{~m}$ in $15 \mathrm{~min}$; at a dilution of $1 / 100$ it did not neutralize phage 5504'. 5504' antiserum, at a dilution of $1 / 1000$, neutralized $90 \%$ of phage 5504' in 15 min., and at a dilution of $1 / 100$ neutralized about $50 \%$ of both phages 187 and $187 \mathrm{~m}$. Since preparations of phage 5504' used for immunization were made by propagating the phage on Ps 187 (which is itself lysogenic for phage $187^{\prime}$ ), all such preparations will contain some phage $187^{\prime}$ as a contaminant, and antisera prepared against phage 5504' will necessarily contain antibodies to phage $187^{\prime}$ and to the serologically identical phage 187. It seems most likely therefore that the cross-reaction observed when phage 187 was exposed to antiserum against phage 5504' was due to these antibodies and that the two phages, 187 and 5504', are serologically distinct.

Table 1. Host ranges of the three phages, 187, $187 m$ and 5504'

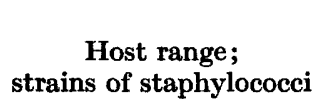

PS 187

5504 unheated

5504 heated

PS 187(187) unheated

PS 187(187) heated

Ps 187(5504') unheated

Ps 187(5504') heated

$\overbrace{187 \quad \begin{array}{c}187 m \\ \text { Efficiency of plating* }\end{array}}^{\text {P504, }}$

$\begin{array}{lll}1 & 1 & 1 \\ 10^{-7} & 10^{-1} & - \\ 10^{-4} & 10^{-1} & - \\ - & - & 1 \\ \overline{10} & - & 10^{-7} \\ 10^{-4} & 10^{-1} & -\end{array}$

* Efficiency of plating on strain Ps 187 as 1.

PS 187 lysogenized with phage 5504' (= PS 187(5504')) was insensitive to phage 187; however, after heating, it was lysed by phage 187 with an eop of $10^{-4}$. Phage 187 grown in these heated cocci was capable of lysing unheated PS 187(5504') and unheated strain 5504 cocci. This strain therefore behaved in the same way as strain 5504, and carriage of phage 5504' appeared to be responsible for the initial untypability of the strain. 
Although carriage of phage 5504' by Ps 187 rendered the strain resistant to both phage 5504' and phage 187, the reverse was not true. When Ps 187 was lysogenized with phage 187 it became immune to phages 187 and $187 \mathrm{~m}$ but remained fully sensitive to phage 5504'. Resistance to phage 187 in strains carring phage 5504' resembled prophage interference as defined by Bertani (1958). The host ranges of the three phages, $187,187 \mathrm{~m}$ and $5504^{\prime}$ are summarized in Table 1.

The results so far reported suggested that carriage of phage 5504' was exerting interference against phage $\mathbf{1 8 7}$ and that this interference was diminished by heat. It seemed possible that heating inactivated prophage 5504' in a proportion of cocci, thus allowing multiplication of phage 187. If this were so it should have been possible to demonstrate non-lysogenic cocci amongst the survivors after heating. Repeated attempts to do this were unsuccessful. Furthermore, although loss of prophage seemed a possible explanation for the results obtained with strain 5504, it could not explain the results with Ps $187\left(5504^{\prime}\right)$, since the phage 187 produced in these cocci was indistinguishable from phage $187 \mathrm{~m}$ in host range. If the cocci producing $187 \mathrm{~m}$ in the PS $187\left(5504^{\prime}\right)$ culture were non-lysogenic, phage 187 should not have undergone any modification, i.e. the phage produced should have been the same as phage 187 grown in PS 187 . The presence of phage 5504' in the productive cocci therefore appeared to be essential for the modification of phage 187. It was concluded that the effect of heating was not to inactivate prophage 5504'.

\section{The inducing effect of heat treatment}

Since loss of prophage 5504' in strains 5504 or PS 187(5504') could not explain the increased sensitivity of the strains to phage 187, the possibility that heat treatment induced the active multiplication of phage 5504' in the lysogenic cocci was investigated. A broth suspension of strain 5504 was divided into two portions, one of which was held at $56^{\circ}$ for $2 \mathrm{~min}$. and rapidly cooled. Both suspensions were then diluted $1 / 100$ in fresh broth and incubated at $37^{\circ}$ for $6 \mathrm{hr}$. At 2-hourly intervals samples were removed and assayed for viable cocci and for free particles of phage 5504 $^{\prime}$ in the supernatant fluids after centrifugation. At the same time the undiluted suspensions were tested for sensitivity to phage 187. Figure 1 shows the result of this experiment. It can be seen that the viable count fell by $76 \%$ as a result of heat treatment, while the free phage count fell by $97 \%$. During the $6 \mathrm{hr}$. incubation period the viable count in the unheated culture rose logarithmically after an initial lag period. The concentration of free phage rose from $1 \times 10^{5} / \mathrm{ml}$. to $5 \times 10^{6} / \mathrm{ml}$. during the $6 \mathrm{hr}$. period, with an average coccus : phage ratio of $60: 1$.

In the heated suspension the viable count remained approximately stationary. The free phage concentration, however, increased from $3 \times 10^{3}$ to $1 \cdot 3 \times 10^{6}$ so that after $6 \mathrm{hr}$. the coccus:phage ratio was about $1: 1$. This represented a 450-fold increase in free phage, whereas the increase in the unheated control culture was only 50-fold. The amount of free phage produced by the heated cocci compared with that produced by unheated cocci provided evidence that heat treatment was inducing active multiplication of the temperate phage in the lysogenic culture.

Sensitivity to phage 187 was about 1000 times greater in the heated suspensions than in the unheated ones, but in both cases sensitivity increased during the $6 \mathrm{hr}$. period. The eop on the heated cocci increased from $5 \times 10^{-5}$ to $2 \times 10^{-4}$ but the eop on the unheated cocci, although only $5 \times 10^{-9}$ at the start of the experiment, 
was $4 \times 10^{-7}$ after incubation for $6 \mathrm{hr}$. It appeared therefore that ageing of the culture alone was responsible for some increase in sensitivity of the cocci to phage 187. This was confirmed by a further experiment in which a broth culture of strain 5504 was held at $37^{\circ}$ for $30 \mathrm{hr}$. and tested for sensitivity to phage 187 after incubation for 6, 24 and $30 \mathrm{hr}$. The eop of the phage at these times was, respectively, $3 \times 10^{-6}, 4 \times 10^{-6}$ and $1.4 \times 10^{-5}$.

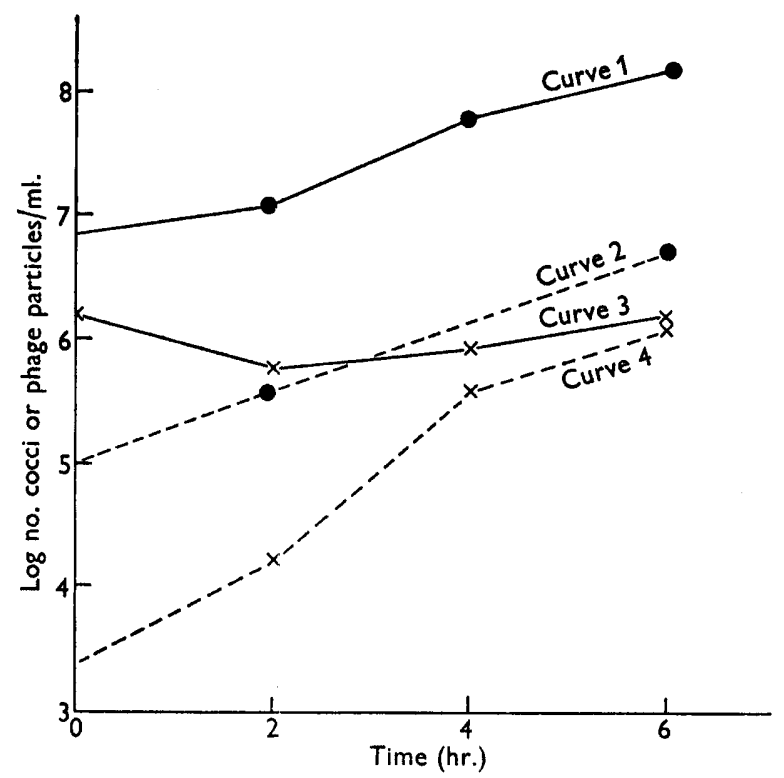

Fig. 1. Growth of organism and production of free phage in strain 5504 after no heat treatment and after exposure to $56^{\circ}$ for $2 \mathrm{~min}$. Curve $1=$ count of cocci in unheated suspension; curve 2 = free phage produced by the unheated culture; curve $3=$ count of cocci in heated suspension; curve $4=$ free phage produced by heated suspension.

\section{Production of phage $187 \mathrm{~m}$ in doubly infected cocci}

Since heat treatment had an inducing effect on the lysogenic strain and since such treatment was known to increase the proportion of cocci which phage 187 could infect, leading to production of phage $187 \mathrm{~m}$, it seemed probable that these two effects were correlated, i.e. that active multiplication of phage 5504' was necessary for the production of phage $187 \mathrm{~m}$. This seemed likely in view of Christensen's results with Shigella dysenteriae strain Sh lysogenic for phage P 1 (Christensen, 1961). In this strain phage $T 1$ undergoes a host-controlled modification. Christensen's results indicated that lysogenic organisms which produced modified phage $\mathbf{T} 1$ were those in which active multiplication of phage $P 1$ was taking place, and that some event dependent upon vegetative multiplication of the carried phage was responsible for the modification of the infecting phage. Experiments were made to see whether the same situation existed here. PS 187 and 187(5504') were used, since all the evidence suggested that PS 187(5504') behaved in exactly the same way as strain 5504. Each of the two strains was infected first with phage 5504' and then with phage 187. Titrations were made during the latent period to count 


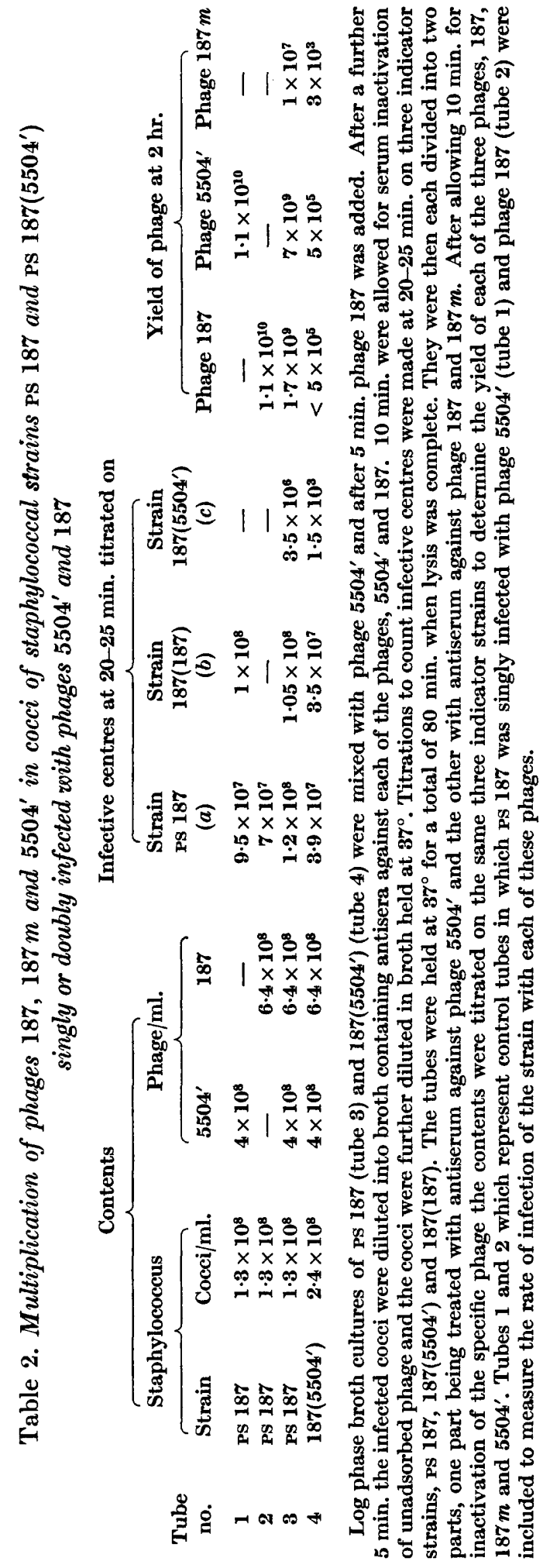


infective centres and again, when lysis was complete, to determine the yield of the three phages, $187,187 m$ and 5504 .

Three indicator strains were used, namely PS 187, PS 187(187) and PS 187(5504'). The sensitivities of these strains are shown in Table 1. PS 187 was fully sensitive to phage $187,187 \mathrm{~m}$ and $5504^{\prime}$ and all infective centres and free phage particles produced a plaque on this strain; Ps $187\left(5504^{\prime}\right)$ was sensitive only to phage $187 \mathrm{~m}$; PS 187(187) was sensitive only to phage 5504'. The difference between the counts on PS 187 and PS 187(187) represented cocci which released only phage 187. It was not possible to distinguish cocci which released only phage 5504' from cocci which released phage 187 and phage 5504', since no strain sensitive only to phage 187 was available. Control tubes were therefore included containing Ps 187 infected separately with each of the two phages, 187 and 5504', and the number of doubly infected cocci was calculated from the figures obtained in these tubes, assuming that a coccus infected with phage 5504' was as susceptible to infection with phage 187 as was an uninfected coccus. Experiments had shown that infection of PS 187 with phage 5504' did not affect the adsorption rate of phage 187.

It was expected that if active multiplication of phage 5504' were essential for the production of phage $187 \mathrm{~m}$, the amount of modified phage would be greatest where Ps 187 was first infected with phage 5504' and subsequently with phage 187, since, in these cocci, phage 5504' would be actively multiplying. Where Ps $187\left(5504^{\prime}\right)$ was exposed to phage 5504' and then to phage 187, only the occasional cocci which underwent spontaneous induction. would be expected to produce phage $187 \mathrm{~m}$, unless superinfection of the strain with phage 5504' had the effect of inducing active multiplication of the carried phage. The results of this experiment, shown in Table 2, can be summarized as follows. Tube 1: in Ps 187 infected with phage 5504' the proportion of cocci infected $\left(1-e^{-x}\right)$ was 0.75 , and the mean burst size (mbs) was 118 phage particles. Tube 2: in Ps 187 infected with phage 187 the proportion of cocci infected $\left(1-e^{-y}\right)$ was 0.54 and the mbs was 157 phage particles. Tube 3 : with PS 187 first infected with phage 5504' and then with phage 187 the platings at 20-25 min. allowed the following figures to be deduced. Total infective centres = $1.2 \times 10^{8}($ column $a)$ : cocci releasing phage $5504^{\prime}=1.05 \times 10^{8}($ column $b) ;$ doubly infected cocci, calculated from the Poisson formula $=5 \cdot 2 \times 10^{7}$; cocci releasing only phage 187 (column $a-$ column $b$ ) $=1.5 \times 10^{7}$; cocci releasing only phage 5504' $\left(1.05 \times 10^{8}-5.2 \times 10^{7}\right)=5.3 \times 10^{7}$; cocci releasing phage $187 \mathrm{~m}=3.5 \times 10^{6}$ (column c) or $6.7 \%$ of the doubly infected cocci. It was not possible to determine from the figures obtained from the 20-25 min. platings whether doubly infected cocci released phage 187 as well as phage 5504', since an indicator strain sensitive to phage 187 and resistant to phage 5504' was not available. However, the yield of phage 187 in tube 3 at $2 \mathrm{hr}$. was only $1.7 \times 10^{9}$. On the assumption that all cocci infected with phage 187, regardless of whether or not they were also infected with phage 5504', released some phage 187, the mbs for phage 187 in tube 3 was only 25 particles. The mbs for phage 187 in tube 2 was 157 particles. If the cocci in tube 3 which released only phage $187\left(1.5 \times 10^{7}\right)$ released a normal burst, these cocci alone could account for all the phage $\mathbf{1 8 7}$ produced in this tube. It is possible that doubly infected cocci released a small burst of phage 187, since the yield of phage $187 \mathrm{~m}$ by those cocci that released any phage $187 \mathrm{~m}$ was only three particles per coccus. A similar yield of unmodified phage 187 would not have been detected. The mbs 
for phage 5504' in tube 3 was 66 particles as compared with 113 in tube 1. This suggested that adsorption of phage 187 to cocci infected with phage 5504' had a 'depressor effect' on the multiplication of phage 5504' (Delbrück, 1945). Tube 4: Ps 187(5504') infected first with phage 5504' and then with phage 187. The figures obtained showed no detectable multiplication of phage 187. Phage 5504' cannot multiply in PS 187(5504') because of the immunity provided by the carried phage. The apparent increase in phage 5504' in this tube could be shown to be due to spontaneous lysis, since an identical culture to which no phage was added produced a similar number of plaques when plated on PS 187 and 187(187). Phage $187 \mathrm{~m}$ was produced by $1.5 \times 10^{3}$ cocci as compared with $3.5 \times 10^{6}$ in tube 3 . When PS $187\left(5504^{\prime}\right)$ was infected only with phage 187 , without superinfection with phage 5504 , the number of cocci producing phage $187 \mathrm{~m}$ was of the same order.

In general, these results are like those described by Christensen (1961) for a Shigella system, the only difference being that, in his system, phage $\mathbf{P} \mathbf{1}$, which exerted interference against phage $\mathbf{T} 1$ when in the prophage state, did not do so when it was in the vegetative state, so that organisms doubly infected with phage $\mathbf{P} 1$ and $\mathbf{T} 1$ produced unmodified, as well as modified, phage $\mathbf{T} 1$. The results presented here and the results of similar experiments with phages 5504' and 187 provided evidence that phage 5504' exerts interference against phage 187, both when the former is in the prophage state and when it is multiplying vegetatively. In both cases, however, the amount of modified phage produced was considerably higher when a non-lysogenic culture was doubly infected with the two phages than when the corresponding lysogenic strain was so treated. The small amount of $187 \mathrm{~m}$ produced by infecting PS $187\left(5504^{\prime}\right)$ with the two phages probably occurred in cocci undergoing spontaneous lysis. We conclude, therefore, that actively multiplying phage 5504' is essential for the production of phage $187 \mathrm{~m}$.

Since the amount of $187 \mathrm{~m}$ produced in strains lysogenic for phage 5504' was dependent on the presence of actively multiplying phage 5504' and since heat treatment induced active multiplication of the carried phage, it is concluded that it is the inducing action of heat which increases the sensitivity of strain 5504 to phage 187.

\section{The effect of ultraviolet irradiation}

Ultraviolet (u.v.) irradiation is known to have an inducing effect on many lysogenic bacteria (Lwoff, Siminovitch \& Kjeldgaard, 1950). Strain 5504 was exposed for varying lengths of time to radiation of wave length $2537 \AA$. from a $15 \mathrm{~W}$. lowpressure germicidal lamp at $17 \mathrm{~cm}$. from the u.v. source. We did not observe any induction of the cultures but the treatment increased the sensitivity of the strain to phage 187. Agar plates, flooded with a broth culture of strain 5504 were irradiated for 10 sec. and phage 187 was then titrated on them. The eop of the phage was $66.0 \times 10^{-7}$ as opposed to $3 \cdot 3 \times 10^{-7}$ on the unirradiated controls. A similar effect of u.v. irradiation was described by Bertani \& Weigle (1953) on $E$. coli strain S. On this strain phage $\lambda C$ plates with a very low eop and the plaques that are produced represent a host-induced modification of the phage. These authors showed that irradiation of strain $S$ increased the eop of phage $\lambda C$ on the strain, and suggested that irradiation changed the physiological condition of the organisms. 


\section{DISCUSSION}

The experiments described show that the untypability of the collection of strains received from Professor Monaci was due to interference exerted by a carried phage, of which phage 5504' has been selected as the representative, against the typing phage, 187. This interference was partly overcome by heating the inocula at $56^{\circ}$ for $2 \mathrm{~min}$., which allowed multiplication of phage 187 in a small proportion of the cocci. It was first considered that the action of heat might be to eliminate the carried phage in a few cocci and thus to overcome the interference. However, this possibility was ruled out for two reasons : (i) no non-lysogenic cocci were detected in heated cultures; (ii) phage 187 produced in heated cocci was modified in its host range in such a way that it was capable of lysing unheated cocci. This modified phage was produced not only in strain 5504, but also in PS 187 lysogenized with phage 5504'. In the latter strain any non-lysogenic cocci would be expected to produce unmodified phage 187 . Since only modified phage 187 was produced in PS 187(5504') after heating, the effect of heating could not have been to eliminate phage 5504'. The evidence suggested that active multiplication of the carried phage was essential for the production of a modified phage $187(187 \mathrm{~m})$. In view of this, phage $187 \mathrm{~m}$ might be expected to represent a genetic recombinant between phage 5504' and 187. However, the phage $187 \mathrm{~m}$ reverted to typical phage 187 after growth in PS 187 , a finding consistent with its being an example of host-induced modification rather than a host-range mutant of phage 187, or a genetic recombinant of phage 5504' and 187. Phage $187 \mathrm{~m}$ differed from the original phage 187 only in its ability to grow in unheated cocci lysogenic for phage 5504'; it was identical serologically and in plaque morphology with phage 187 .

Heat treatment of strains lysogenic for phage 5504' had a slight inducing effect and it is suggested that this induction is responsible for the increased sensitivity of the strains to phage $18 \%$, since it would provide more cocci containing actively multiplying phage 5504', and thus more cocci in which phage $187 \mathrm{~m}$ could be produced. It is possible that induction by heat is brought about by destroying a cytoplasmic repressor substance which, it is suggested, is responsible for maintaining the stability of the prophage state in the strain (Jacob \& Campbell, 1959). Treatment with u.v. radiation also increased the sensitivity of the strains to phage 187 . Perhaps heat treatment and u.v. irradiation both produce their effects by destroying a cytoplasmic repressor substance.

The increase in the eop of phage 187 from $10^{-7}$ to $10^{-4}$ as a result of heat treatment was sufficiently great to enable these epidemiologically related strains to be recognized as being identical. Since $1000 \mathrm{rtd}$ preparations of phages used in routine typing of staphylococci contain about $10^{8}$ particles $/ \mathrm{ml}$., the strains were not lysed, and would not have been recognized as belonging to the phage type had they not been treated by heat. It is possible that, where a host-induced modification of the typing phages can take place, a similar effect may be observed on other strains of staphylococci, which would increase the strength of the reactions and thereby widen the spectrum of lysis. It is questionable, however, whether such an expansion of the lytic spectrum would have any practical value except in special cases such as the one described here, where a strain would otherwise be untypable. The effect of heating was investigated on two other strains of staphylococci. One 
of these strains, previously not sensitive to phage 80 , became sensitive after heating, and with the other strain the same effect was demonstrated with phage 71 . In both cases the phages grown in the heated strains had been modified in that they now lysed unheated cocci of the same strain. This was not investigated further; the phenomenon does not appear to be uncommon.

\section{REFERENCES}

Adams, M. H. (1950). Methods of study of bacterial viruses. Meth. med. Res. 2, 1.

Anderson, E. S. \& Felix, A. (1952). Variation in Vi-phage II of Salmonella typhi. Nature, Lond. 170, 492.

Anderson, E. S. \& Felix, A. (1953). The Vi-type determining phages carried by Salmonella typhi. J. gen. Microbiol. 9, 65.

Bertani, G. (1958). Lysogeny. Advanc. Virus Res. 5, 161.

Bertant, G. \& Weigle, J. J. (1953). Host-controlled variation in bacterial viruses. J. Bact. 65, 113.

Christensen, J. R. (1961). On the process of host-controlled modification of bacteriophage. Virology, 13, 40.

DeLBRücK, M. (1945). Interference between bacterial viruses. III. Mutual exclusion and the depressor effect. J. Bact. 50, 151.

JAcob, F. \& CAmpbell, A. (1959). Sur le système de répression assurant l'immunité chez les bactéries lysogènes. C.R. Acad. Sci., Paris, 248, 3219.

Lederberg, J. \& Lederberg, E. M. (1952). Replica plating and indirect selection of bacterial mutants. J. Bact. 63, 399.

Luria, S. E. (1953). Host induced modifications of virus. Cold. Spr. Harb. Symp. quant. Biol. 18, 287.

LuRIA, S. E. \& Human, M. L. (1952). A non-hereditary host induced variation of bacterial viruses. J. Bact. 64, 557 .

Lwoff, A., Siminovitch, L. \& KJeldgaArd, N. (1950). Induction de la production de bactériophages chez une bactérie lysogène. Ann. Inst. Pasteur, 79, 815.

Ma, P. \& MANdLe, R. J. (1961). Phage typing of non-typable strains of Staphylococcus aureus. Bact. Proc. p. 111.

Muzzetto, M., Calonghx, G. F., Zorzi, M., Dossena, G., Sueri, L. \& Vitali, M. A. (1961). Descrizione di un grave episodio epidemico in comunità chiusa da Stafilococco 187. G. Mal. infett. 13, 765.

Ralston, D. J. \& Krueger, A. P. (1952). Phage multiplication in two hosts. Isolation and activity of staphylococcus phage P1. Proc. Soc. exp. Biol., N.Y. 80, 217.

Swanstrom, M. \& Adams, M. H. (1951). Agar layer method for production of high titre phage stocks. Proc. Soc. exp. Biol., N.Y. 78, 372.

WAHL, R. \& FouACE, J. (1954). Isolement et emploi de phages nouveaux pour identifier les souches de staphylocoques pathogènes insensibles aux phages classiques. Ann. Inst. Pasteur, 86, 161. 